\title{
Characterization of DAPK1 as a novel transcriptional target of BRMS1
}

\author{
${\text { XIAOJING } \text { QIAO }^{1 *}, \text { XI YANG }^{1 *}, \text { YIREN ZHOU }^{1}, \text { XINYU MEI }^{1}, \text { JIANMING DOU }^{1} \text {, WENJUAN XIE }}^{1}$, \\ GUOQING LI $^{1}$, YEKAI WANG ${ }^{1}$, SHOUYI QIAO ${ }^{1}$, JIANWEI HU ${ }^{2}$ and YANHUA WU ${ }^{1}$ \\ ${ }^{1}$ State Key Laboratory of Genetic Engineering, Institute of Genetics, School of Life Sciences, \\ Fudan University, Shanghai 200433; ${ }^{2}$ Endoscopy Center and Department of General Surgery, \\ Zhongshan Hospital of Fudan University, Shanghai, 200032, P.R. China
}

Received December 28, 2016; Accepted February 23, 2017

DOI: 10.3892/ijo.2017.3930

\begin{abstract}
Breast cancer metastasis suppressor 1 (BRMS1) can specifically regulate tumor metastasis in many cancers. Our previous studies have demonstrated that BRMS1 can promote cell apoptosis through regulating osteopontin (OPN) expression in hepatocellular carcinoma (HCC) cells. However, the transcriptional targets of BRMS1 have not been thoroughly studied. In this study, death-associated protein kinase 1 (DAPKI), a tumor suppressor gene with multiple roles in regulating cell death, was identified as a potential transcriptional target of BRMS1 in the whole genome expression microarray. Quantitative real-time PCR and western blot analysis of HCC cells overexpressing BRMS1 further confirmed the transcriptional regulation relationship between BRMS1 and DAPK1. Moreover, DAPK1 expression was frequently decreased or even lost in HCC tissue samples by comparison with neighboring pathologically normal liver tissue, which was consistent with the decreased BRMS1 expression pattern. To unravel the molecular mechanism of BRMS1 in regulating DAPK1, a series of deletion mutants of $D A P K 1$ promoter was subjected to luciferase assay. The luciferase units of -200 to $-80 \mathrm{bp}$ region, with two tandem putative NF- $\kappa \mathrm{B}$ binding sites, were specifically enhanced by BRMS1 expression. Site-directed mutants of NF- $\mathrm{KB}$ binding sites blocked the transcriptional activation effect. In addition, the binding capability of BRMS1 and the putative NF- $\mathrm{\kappa B}$ binding
\end{abstract}

Correspondence to: Professor Yanhua Wu, School of Life Sciences, Fudan University, Shanghai 200433, P.R. China

E-mail: yanhuawu@fudan.edu.cn

Dr Jianwei Hu, Endoscopy Center and Department of General Surgery, Department of General Surgery, Zhongshan Hospital of Fudan University, Shanghai 200032, P.R. China

E-mail: jwhoo@hotmail.com

${ }^{*}$ Contributed equally

Key words: hepatocellular carcinoma, breast cancer metastasis suppressor 1, death-associated protein kinase 1, transcriptional regulation sites were demonstrated in the chromatin immunoprecipitation (ChIP) assay. In conclusion, our study characterized DAPKI as a novel transcriptional target of BRMS1. Transcriptional activation of DAPK1 might be another important mechanism accounting for the metastasis suppressive activity of BRMS1.

\section{Introduction}

Hepatocellular carcinoma (HCC) is among the most common and highly lethal cancers worldwide, with a depressingly low long-term survival rate (1). So far, tumor metastasis remains the primary cause of death for most HCC patients. Tumor metastasis consists of several discrete biological processes, initiating from escaping primary tumor site, invading and surviving in the surrounding tissues, entering the lymphatic vessels or the bloodstream, eventually transporting to a remote site and forming new colonies (2).

Breast cancer metastasis suppressor 1 (BRMS1) is an active tumor metastasis suppressor gene, exhibiting tumor metastasis suppressive activity in breast cancer, melanoma and non-small cell lung cancer (3-5). Many studies have reported that the expression of BRMS1 was silenced due to gene mutation or promoter hypermethylation in metastatic tumor cells (6-8). Functional studies revealed that BRMS1 was involved in the regulation of cell-cell communication, cell migration, cell invasion, cell apoptosis and tumor angiogenesis, while no obvious effect has been shown on cell proliferation, cell-matrix adhesion and matrix degradation (9). Mechanistically, BRMS1 is an essential part of mSin3a.HDAC complex, which modulates gene transcription activity by regulating the acetylation levels of both histone and transcriptional factors (10). Therefore, several cancer-related genes have been recently characterized as transcriptional targets of BRMS1, including CXCR4, $c L A P-2, B c l-x L, u P A$ and miR146 (9). We have previously demonstrated that BRMS1 was able to sensitize HCC cells to apoptosis through suppressing NF- $\kappa \mathrm{B}$ signaling pathway and osteopontin expression $(11,12)$.

In this study, we started from gene expression microarray and identified a novel BRMS1 target gene, death-associated protein kinase $1(D A P K 1)$, in HCC cells. DAPK1 is a well-defined tumor suppressor gene, with significant suppressive effect in both tumor growth and metastasis in vivo (13). DAPK1 
participates in multiple cell death-related signaling pathways, including caspase-dependent cell apoptosis, mitochondrialdependent cell apoptosis and autophagic cell death (14). In a variety of tumor tissues and cell lines, the promoter region of DAPK1 was hypermethylated, resulting in significant decrease or loss of $D A P K 1$ expression $(15,16)$. Additionally, many transcriptional factors including TP53, C/EBP- $\beta$, HSF1 and SMAD can transcriptionally activate $D A P K 1$ expression, while STAT3 and NF- $\kappa$ B play the opposite role (17).

We reported for the first time that BRMS1 could transcriptionally activate DAPK1 expression in HCC cells. Immunohistochemical analysis of human HCC tissues revealed that $D A P K 1$ expression was specifically silenced in tumor cells. The association relationship between BRMS1 and $D A P K 1$ expression in paired HCC tissues was studied through western blot analysis and the transcriptional mechanism of BRMS1 on DAPK1 promoter was further elucidated. Our findings suggested a functional relationship between BRMS1 and DAPK1, indicating another potential molecular mechanism accounting for BRMS1's tumor suppressive role in HCC cells.

\section{Materials and methods}

Ethics statement. This study was accomplished with the approval of the Medical Ethics Committee of School of Life Sciences, Fudan University, Shanghai, China.

Tumor specimens. Fresh surgical specimens of HCC, including tumor tissues and the neighboring pathologically non-tumorous liver tissues, were obtained from liver cancer patients at Zhongshan Hospital (Fudan University, Shanghai, China). All of the samples were immediately frozen in liquid nitrogen after surgery and then stored at $-80^{\circ} \mathrm{C}$ before further analysis.

Tissue microarray analysis. Fifty matched pairs of tumor samples and adjacent normal tissues from clinical HCC patients were used for the construction of a tissue microarray (Shanghai Biochip Co., Ltd. Shanghai, China) as previously described (18). In brief, sections (4 $\mu \mathrm{m}$ thickness, $2 \mathrm{~mm}$ diameter) were taken from individual paraffin-embedded tissues and precisely arrayed on 3-aminopropyltriethoxysilane-coated slides for subsequent staining with an anti-DAPK1 antibody (Sigma, USA). The immunohistochemistry analysis was performed in Shanghai Biochip. All the images were visualized by Leica DC 500 camera on a microscope equipped with Leica DMRA2 fluorescent optics (LEICA).

Quantitative real-time PCR ( $q R T-P C R)$. Total RNA was extracted from tissues or cultured cells using TRIzol reagent (Life Technologies, USA), and 1-2 $\mu \mathrm{g}$ of RNA was used for reverse transcription using PrimeScript ${ }^{\mathrm{TM}} \mathrm{RT}$ reagent kit with gDNA Eraser (Takara, Japan). PCR analysis was performed using the SYBR Green Supermix kit (Takara) with the CFX Connection detection system (Bio-Rad, USA). Diluted cDNA was used in a $10-\mu 1$ real-time PCR reaction in triplicate for each gene and each sample. Cycle parameters were $95^{\circ} \mathrm{C}$ for $5 \mathrm{~min}$ hot start and 40 cycles of $95^{\circ} \mathrm{C}$ for $5 \mathrm{sec}, 58^{\circ} \mathrm{C}$ for $10 \mathrm{sec}$ and $72^{\circ} \mathrm{C}$ for $20 \mathrm{sec}$. Blank controls with no cDNA templates were performed to rule out contamination. The specificity of the PCR product was confirmed by melting curve analysis.
BRMS1 primers were: forward, 5'-ACTGAGTCAGCTGC GGTTGCGG-3'; reverse, 5'-AAGACCTGGAGCTGCCTC TGGCGTGC-3'. DAPK1 primers were: forward, 5'-TGTCTT CCACCAACTCCAGCAG-3'; reverse, 5'-AAATCGCCAAC TCCATTCAAATAAGC-3'. 18S $r R N A$ primers were: forward, 5'-GTAACCCGTTGAACCCCATT-3'; reverse, 5'-CCATCCA ATCGGTAGTAGCG-3'.

The expression levels of all genes were normalized to those of the house keeping gene $18 \mathrm{~S} r R N A$. Relative gene expression levels were calculated by the formula $2^{-\Delta \mathrm{Ct}}$, where $\Delta \mathrm{Ct}$ (Critical threshold $)=\mathrm{Ct}$ of genes of interest $-\mathrm{Ct}$ of $18 \mathrm{~S} r R N A$.

Plasmid construction. Full length of DAPK1 promoter was amplified from human genomic DNA, forward, 5'-CACTCACT CCCTAGCTGTGT-3'; reverse, 5'-TAGCCCCCTCATGCA-3'. The amplicons were separated by DNA electrophoresis and purified before being cloned into the pGEM-T Easy Vector (Promega, USA). Both the full-length promoter and three 3' deletion mutants were further cloned into pGL3-Basic Vectors (Promega). The relevant primers were as follows: pGL3-Basic-DAPK1-P(P): forward, 5'-GGGGTACCCACT CACTCCCTAGCTGTGT-3'; reverse, 5'-CCAAGCTTTAGCC CCCTCATGCA-3'. pGL3-Basic-DAPK1-P1(P1): forward, 5'-GGGGTACCCACTCACTCCCTAGCTGTGT-3'; reverse, 5'-CCAAGCTTGACCGGGTCTCCGGA-3'. pGL3-BasicDAPK1-P2(P2): forward, 5'-GGGGTACCCACTCACTCCCT AGCTGTGT-3'; reverse, 5'-CCAAGCTTCCACCTCCAGGG ACG-3'. pGL3-Basic-DAPK1-P3(P3): forward, 5'-GGGGT ACCCACTCACTCCCTAGCTGTGT-3'; reverse, 5'-CCAAG CTTGGCGACTCCCTCTCC-3'. Two site-directed mutations of pGL3-Basic-DAPK1-P, Mut1 and Mut2, were obtained by KOD-Plus-Mutagenesis kit (Toyobo, Japan) according to the manufacturer's instructions. The relevant primers were as follows: Mut1: forward, 5'-TCTGAGCGCCGGGGAGGTCT ACTTCCTTTT-3'; reverse,5'-AACCGCTCGCTGAAGACCG GGTCTCCGGAG-3'. Mut2: forward, 5'-AGGGATACTTCCT TTTGGGGTTGCCATTTT-3'; reverse, 5'-CAACGGCGCTA AGACCCCGCTCGCTGAAGA-3'.Recombinant pCMV-MycBRMS1 were constructed as previously described (11).

Cell culture and transfection. Human embryonic kidney cell line 293T and HCC cell line SK-Hep1 were all cultured with Dulbecco's modified Eagle's medium (DMEM), supplemented with $10 \%$ fetal bovine serum (Gibco, USA) at $37^{\circ} \mathrm{C}$ in $5 \% \mathrm{CO}_{2}$ humidified atmosphere. Cells at $80 \%$ confluency were transfected using Lipofectin 2000 (Invitrogen, USA) according to the manufacturer's instructions.

Dual-luciferase assay. Cells were seeded into 24-well culture plates at a density of $1 \times 10^{5} /$ well and transfected with indicated $D A P K 1$ promoter constructs or pGL3-Basic empty vectors. The pRL-TK control vector (20 ng/well) was used for normalization. Cells were harvested $36 \mathrm{~h}$ after transfection. Firefly and Renilla activities were determined using GloMax 96 Microplate Luminometer (Promega) according to the manufacturer's instructions. Data are presented as the changes in Firefly luciferase activity relative to Renilla luciferase activity.

Western blot analysis. Protein samples were separated by $10 \%$ SDS-PAGE gel and then transferred to PVDF membranes. 
A

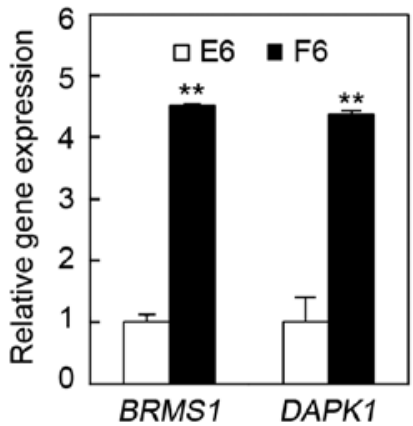

B

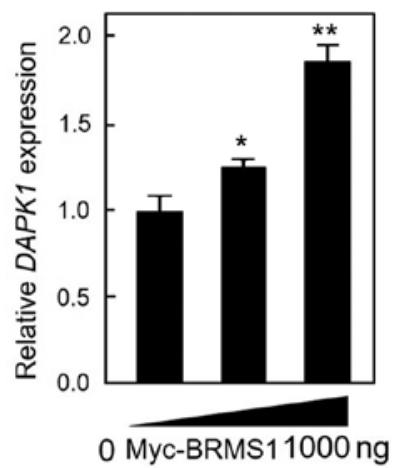

C

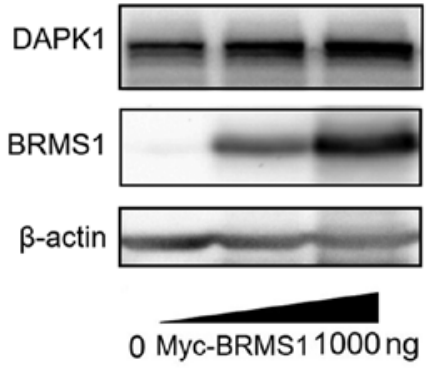

Figure 1. Human DAPK1 was transcriptionally activated by BRMS1. (A) Relative BRMS1 and DAPK1 mRNA expression levels in SK-Hep1 cell clone stably expressing BRMS1 (F6) and control cell clone (E6) were analyzed through gene expression microarray. Values are normalized to internal control and expressed as mean $\pm \mathrm{SD}, \mathrm{n}=3$. (B) Relative DAPK1 expression levels in SK-Hep1 cells transiently expressing different dosages of pCMV-Myc-BRMS1 (Myc-BRMS1) were quantified through qRT-PCR. Values are normalized to internal control $18 \mathrm{~S} r R N A$ and expressed as mean $\pm \mathrm{SD}, \mathrm{n}=3$. (C) Western blot analysis of DAPK1 and BRMS1 protein expression levels in SK-Hep1 cells transiently expressing different dosages of Myc-BRMS1. $\beta$-actin was used as a loading control.

Non-specific binding was blocked by incubation with $5 \%$ fat-free milk for $1 \mathrm{~h}$ at room temperature. After blocking, the membranes were incubated with specific primary antibodies against different proteins at $4{ }^{\circ} \mathrm{C}$ overnight, followed by incubation with HRP-conjugated secondary antibody for $45 \mathrm{~min}$ at room temperature. Immunoreactivity was visualized by enhanced chemiluminescence (Pierce, USA) on a molecular imager ChemiDoc $\mathrm{XRS}^{+}$system (Bio-Rad). Related antibodies included the mouse monoclonal antibody against BRMS1 (Abcam, USA), $\beta$-actin and Myc tag (Sigma), the rabbit monoclonal antibody against DAPK1 (Sigma), peroxidase-conjugated goat anti-mouse IgG and goat anti-rabbit IgG (Jackson, USA).

Chromatin immunoprecipitation (ChIP). The ChIP assay was performed according to Farenham laboratory protocol (19). Basically, HEK 293 T cells were seeded at $1 \times 10^{7} /$ dish in 100-mm dishes and transfected with pCMV-Myc-BRMS1 plasmid or empty pCMV-Myc vector. At $36 \mathrm{~h}$ post-transfection, cells were subject to crosslinking and fixation using $4 \%$ formaldehyde (Amresco, USA). Cell nuclei were released by SDS lysis buffer (1\% SDS, $10 \mathrm{mM}$ EDTA, and $50 \mathrm{mM}$ Tris- $\mathrm{HCl}$, pH 8.1) before sonication. Fragmented genomic DNA was immuno-precipitated by anti-Myc antibody at $4^{\circ} \mathrm{C}$ after precleared with protein A-agarose beads. Mouse IgG was used as the negative control to exclude genomic contamination. The immune-precipitated DNA fragments were then collected, washed, de-crosslinked and digested with Proteinase K. DNA fragments were recovered by phenol/chloroform extraction and amplified through qRT-PCR using the following primers: primers covering the putative $N F-\kappa B$ binding site $(\mathrm{P}-\mathrm{A})$ : forward, 5'-CAGCGAGCGGGGTCTTAG-3'; reverse, 5'-GTA AAATGGCAACCCCAAAA-3'. Primers covering the downstream region of the putative $N F-\kappa B$ binding site $(\mathrm{P}-\mathrm{B})$ : forward, 5'-TCTTCAAAAGGACTGGAGACTGA-3'; reverse, 5'-CCTGCCAAGTTCCTCGCC-3'.

Statistical analysis. Comparisons of quantitative data were analyzed by Student's t-test. Categorical data were analyzed by Fisher's exact test. We considered $\mathrm{p}<0.05$ to be different and $\mathrm{p}<0.01$ to be significant different.

\section{Results}

DAPK1 is positively regulated by BRMS1 in HCC cells. In order to find novel transcriptional targets of BRMS1, BRMS1 stably expressing clone F6 and control clone E6 established in our previous study were analyzed in a whole genome expression microarray (11). Under the selection criteria of |Fold change| >2, 260 potential target genes were found, of which 194 genes were upregulated and the other 66 genes were suppressed by BRMS1 expression (data not shown). A well-studied tumor suppressor gene, DAPK1 was found to be 4.46-fold overexpressed in cells stably expressing BRMS1 by comparison with control cells (Fig. 1A). Next, qRT-PCR and western blot analyses were carried out to confirm this result. Different dosages of recombinant BRMS1 plasmid were transiently introduced into SK-Hep1 cells. As shown in Fig. 1B and $\mathrm{C}$, both the mRNA and the protein expression levels of $D A P K 1$ were, not surprisingly, upregulated upon BRMS1 overexpression in a dose-dependent manner. The data strongly suggest that DAPK1 is a potential transcriptional target of BRMS1 in HCC cells.

DAPK1 is remarkably downregulated in human HCC tissues. To assess the expressional pattern of DAPK1 in HCC tissues, immunohistochemical analysis was carried out on a tissue microarray including 50 paired HCC tissues and adjacent non-tumorous liver tissues. It was revealed that the immunostaining signal of DAPK1 is reduced in 37 tumor tissues by comparison with adjacent non-tumorous tissues, of which, 18 tumor tissues exhibited remarkably suppressed or even silenced DAPK1 expression. Images from four representative HCC samples composed of both tumor cells and normal liver cells are shown in Fig. 2A. In contrast to high DAPK1 expression in normal liver cells, DAPK1 immunostaining signal is totally lost in the surrounding tumor cells. To further confirm the expression pattern of DAPK1 in HCC samples, additional 
A
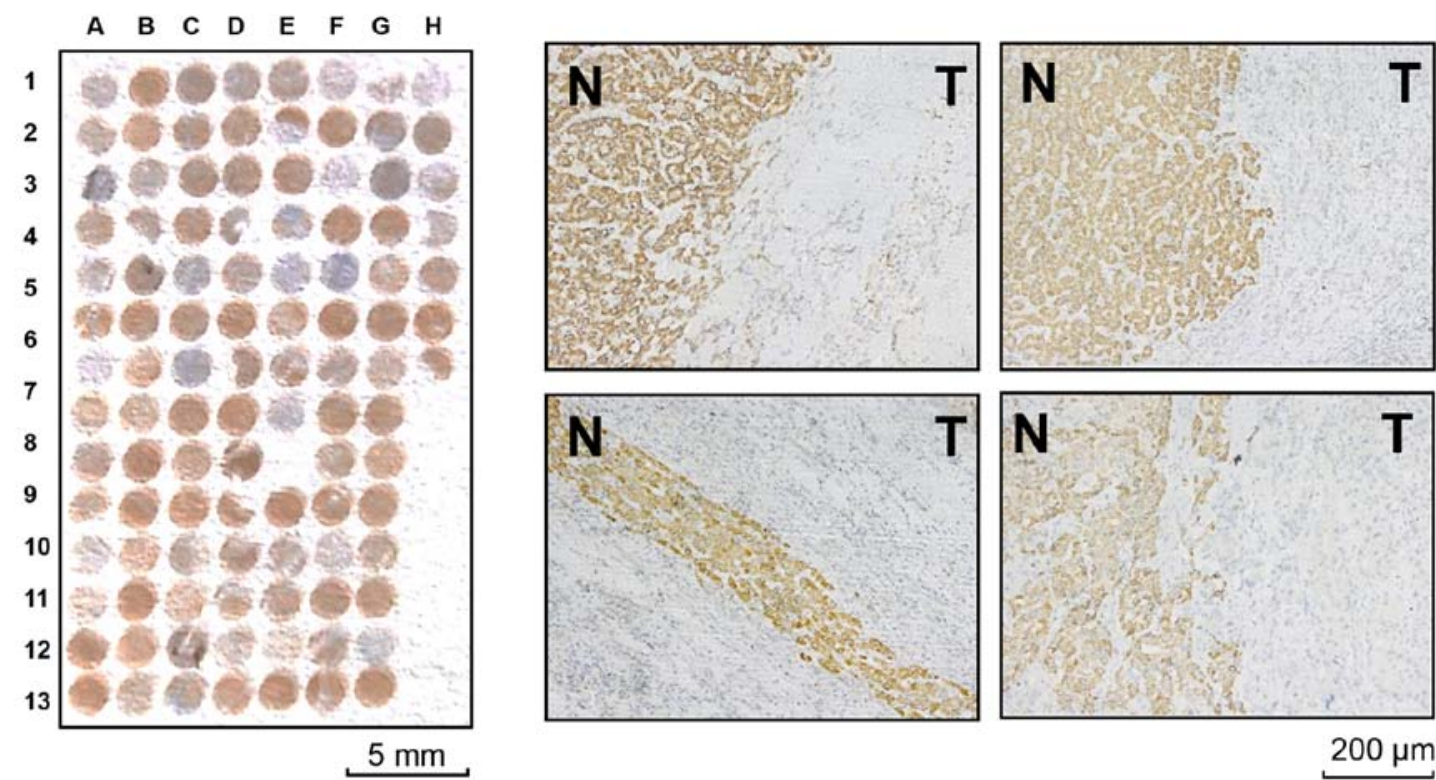

$200 \mu \mathrm{m}$

B

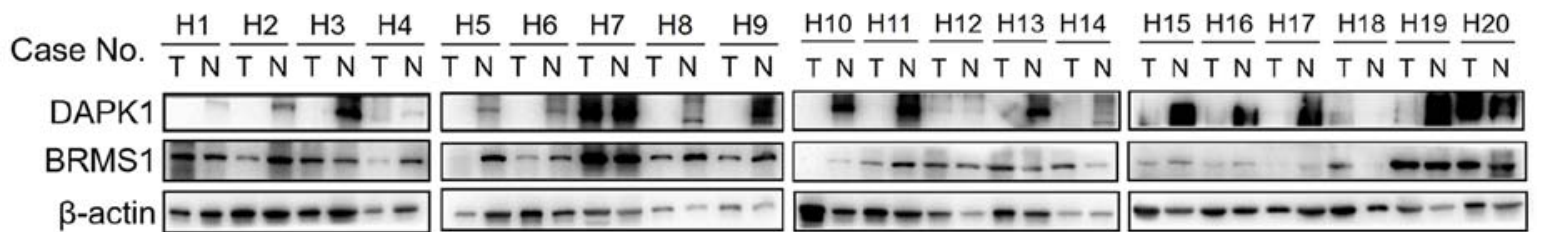

Figure 2. DAPK1 is remarkably downregulated in paired HCC specimens. (A) A tissue microarray composed of 50 paired HCC specimens with corresponding non-tumorous tissues was subjected to immunohistochemical staining using the anti-DAPK1 antibody. Left panel, the full image of the tissue microarray Right panel, representative images derived from four HCC specimens with both tumor cells (T) and normal liver cells (N). (B) Western blot analysis of DAPK1 and BRMS1 protein expression in other 20 paired (case no., H1-H20) HCC specimens (T) with corresponding non-tumorous tissues (N). $\beta$-actin was used as a loading control.

20 paired HCC tissues and adjacent non-tumorous liver tissues were subjected to western blot analysis (Fig. 2B). Consistently, DAPK1 protein expression was almost completely lost in 15 out of 20 tumor tissues. We have previously investigated the BRMS1 expression in these HCC samples (11), whether DAPK1 expression was associated with BRMS1 expression in these paired protein samples needed to be assessed. It was shown that in all 11 samples with downregulated BRMS1 expression, 10 samples exhibited consistent DAPK1 downregulation (positive ratio $=90.91 \%$ ). Moreover, only 5 out of the other 9 samples without suppressed BRMS1 expression exhibited silenced DAPK1 (positive ratio $=55.56 \%$ ). This finding indicates a potential correlation of endogenous BRMS1 and DAPK1 expression in clinical HCC tissues, providing another piece of evidence that DAPK1 might be transcriptionally regulated by BRMS1.

DAPK1 promoter is transcriptionally activated by BRMS1 in a dose-dependent manner. To clarify the transcriptional mechanism between BRMS1 and DAPK1, the promoter region (-1,084 to $-80 \mathrm{bp}$ ) of human $D A P K 1$ gene was cloned from human genomic DNA and a series of 3 ' deletion mutants, namely P1 ( $-1,084$ to $-200 \mathrm{bp}), \mathrm{P} 2$ (-1,084 to $-436 \mathrm{bp})$ and P3 (-1,084 to $-627 \mathrm{bp}$ ), were constructed subsequently (Fig. 3A). The four DNA fragments were then inserted into pGL3-Basic vector to detect their transcriptional activity in the luciferase assay, respectively. As shown in Fig. 3B, by comparison with full length promoter, both pGL3-Basic-P1(P1) and pGL3-Basic$\mathrm{P} 2(\mathrm{P} 2)$ truncates exhibited a $50 \%$ reduction in the report gene's activity, and pGL3-Basic-P3(P3) almost lost the entire transcriptional activity. This result suggests that -200 to $-80 \mathrm{bp}$ and -627 to $-436 \mathrm{bp}$ are two potential transcriptional active regions in $D A P K 1$ promoter. Next, Myc-BRMS1 plasmid was co-introduced into cells to investigate the transcriptional effect of BRMS1 on DAPK1 promoter. It was found that BRMS1 can transcriptionally activate the luciferase activity of pGL3-Basic-P(P), whereas no effect was observed on the other three truncates (Fig. 3C). Moreover, when different dosages of Myc-BRMS1 plasmid were utilized, it was shown that the luciferase activity of pGL3-Basic-P gradually increased upon the expression of exogenous BRMS1 (Fig. 3D). Taken together, current results indicate that BRMS1 might be able to activate $D A P K 1$ expression through the -200 to $-80 \mathrm{bp}$ region of the promoter.

BRMS1 is able to bind the putative $N F-\kappa B$ binding sites of $D A P K 1$ promoter. After identifying -200 to $-80 \mathrm{bp}$ region of $D A P K 1$ promoter as the target of interest, we screened this region using online transcriptional element prediction software to uncover the potential transcriptional factor binding 
A

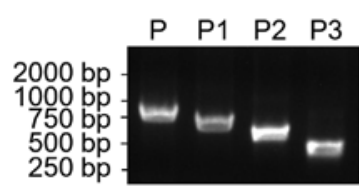

C

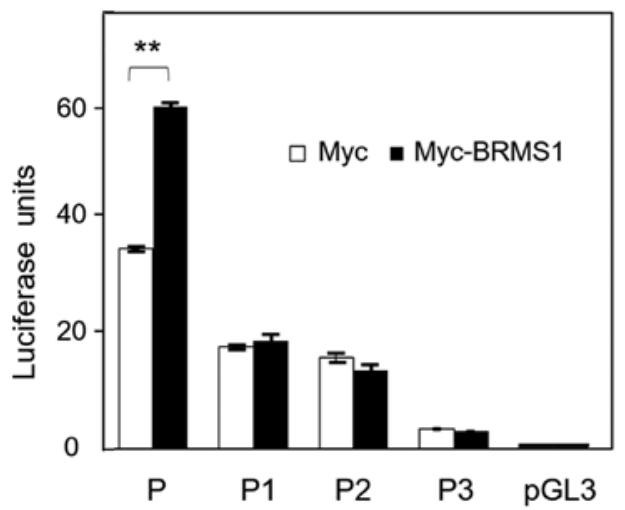

B

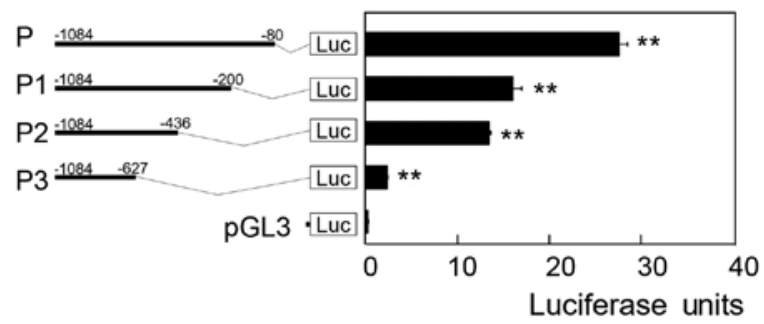

D

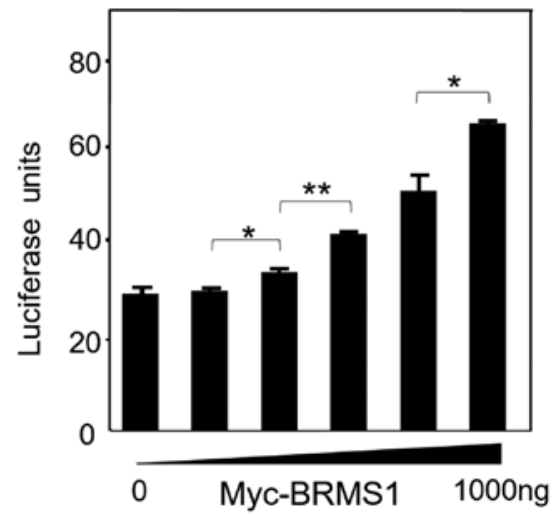

Figure 3. BRMS1 is able to transcriptionally activate DAPK1 promoter's activity. (A) Gel electrophoresis analysis of the full length $D A P K 1$ promoter (P) and three deletion mutants (P1, P2 and P3). (B) Schematic of four recombinant luciferase reporter constructs (left panel) and relative reporter activities in HEK293T cells (right panel). Values are expressed as mean \pm SD, $n=3$. (C) Recombinant Myc-BRMS1 or empty vector was transfected into HEK293T cells together with indicated recombinant luciferase reporter constructs. Reporter activity values are expressed as mean $\pm S D, n=3$. (D) Relative reporter activities were analyzed in cells co-transfected with pGL3-P and different dosages of Myc-BRMS1. Reporter activity values are expressed as mean $\pm \mathrm{SD}, \mathrm{n}=3$. ${ }^{*} \mathrm{P}<0.05 ;{ }^{* *} \mathrm{P}<0.01$.

A

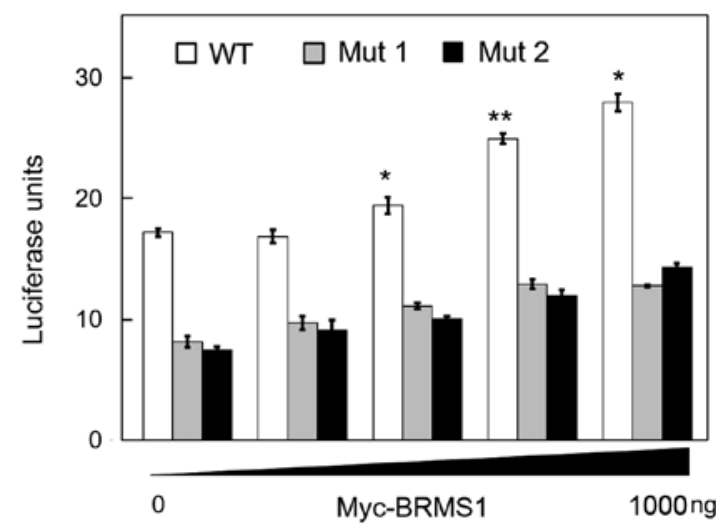

WT : $\quad$ CGgGGTCTTA GCGCCGgGGA GGTCTACTTC

Mut1: CGGTTTCTGA GCGCCGGGGA GGTCTACTTC

Mut2: CGGGGTCTTA GCGCCGTTGA GGGATACTTC
B

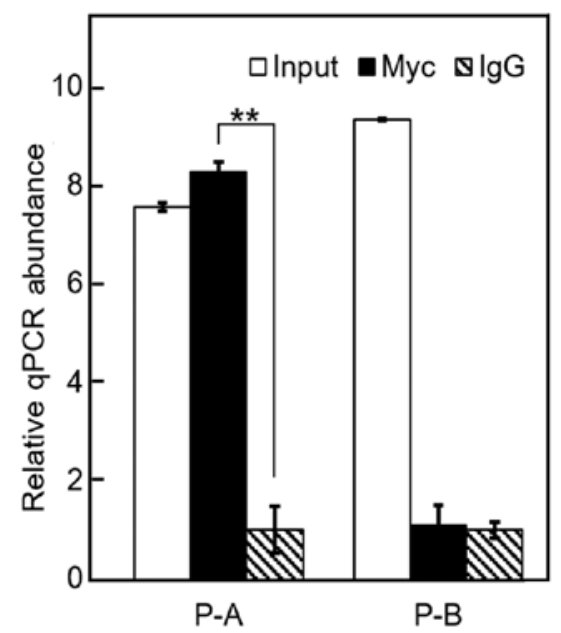

Figure 4. Exogenous BRMS1 binds to the putative NF- $\mathrm{B}$ binding site of DAPK1 promoter. (A) Nucleotide sequences of the putative NF- $\mathrm{B}$ binding sites of wild-type DAPK1 promoter (WT) and two site-directed mutants (Mut1 and Mut2) are shown in the bottom. Mutant nucleotides are underlined and indicated in grey. Relative reporter activities were analyzed in cells co-transfected with indicated reporter constructs and Myc-BRMS1. Values are expressed as mean $\pm \mathrm{SD}, \mathrm{n}=3$. (B) ChIP analysis of the putative NF- $\mathrm{kB}$ binding site in DAPK1 promoter. Soluble chromatin was prepared from HEK293T cells transfected with Myc-BRMS1 and immunoprecipitated by an anti-Myc antibody or IgG control. Two different pairs of primers (P-A, P-B) were utilized in the qRT-PCR analysis. Blank controls with no DNA templates were utilized to rule out contamination and soluble chromatin before immunoprecipitation was utilized as input control. Values are expressed as mean $\pm \mathrm{SD}, \mathrm{n}=3 .{ }^{*} \mathrm{P}<0.05 ;{ }^{* *} \mathrm{P}<0.01$.

sites. The -190 to -181 bp (CGGGGTCTTA) region and the -176 to -167 bp (CGGGGAGGTC) region of DAPK1 promoter were predicted to be two tandem putative binding sites for transcription factor NF- $\mathrm{KB}$, which is demonstrated to be an important deacetylation modification target of BRMS1 (20-22). To assess whether BRMS1 functions through these binding sites, two site-directed point-mutations of pGL3-Basic-P, Mut1 and Mut2, were constructed. As shown in Fig. 4A, both 
mutations markedly abolished the transcriptional activation effect of BRMS1 on DAPK1 promoter, strongly suggesting that these putative NF- $\kappa \mathrm{B}$ binding sites might be involved in BRMS1-DAPK1 transcriptional regulation. Chromatin immunoprecipitation experiment was further carried out in order to demonstrate whether BRMS1 could bind to this DAPK1 promoter region. Cells overexpressing Myc-BRMS1 were lysed and immunoprecipitated by anti-Myc antibody or IgG control. Two different pairs of primers were designed to target the two putative $\mathrm{NF}-\kappa \mathrm{B}$ binding sites $(\mathrm{P}-\mathrm{A})$ and the downstream region of $\mathrm{NF}-\kappa \mathrm{B}$ binding sites $(\mathrm{P}-\mathrm{B})$, respectively. As shown in Fig. $4 \mathrm{~B}$, only the promoter region containing NF- $\kappa \mathrm{B}$ binding sites was immunoprecipitated by specific antibody against exogenous BRMS1. These data together demonstrate that BRMS1 is able to bind DAPK1 promoter through the putative NF- $\kappa \mathrm{B}$ binding sites.

\section{Discussion}

After BRMS1 was demonstrated to be a functional partner of $\mathrm{mSin} 3 \mathrm{a} \cdot \mathrm{HDAC}$ complex involved in regulating chromatin status and gene expression, several BRMS1-target genes have been demonstrated to be involved in BRMS1-mediated tumor metastasis suppression. Herein, we reported for the first time that DAPK1 was another transcriptional target of BRMS1 in HCC cells. DAPK1 was initially identified as a positive mediator of apoptosis activated by interferon- $\gamma$ in HeLa cell (23). Soon after that, DAPK1 was well demonstrated as an important tumor suppressor, which also functions in suppressing tumor metastasis (24). For example, Inbal et al found that restoration of DAPK1 to physiological levels in high-metastatic Lewis carcinoma cells successfully suppressed tumor metastasis in a mouse model (25). By utilizing in situ TUNEL staining of tumor sections, they proposed that loss of DAPK1 expression provides a unique mechanism that links suppression of apoptosis to metastasis. Kuo et al reported that DAPK1 can block cell migration and invasion in tumor cells by blocking the integrin-mediated polarity pathway (26). Additionally, Chen et al revealed that miR-103/107 promote metastasis of colorectal cancer probably through targeting DAPK1 (27). It is therefore of great interest to uncover the relationship between BRMS1 and DAPK1 in HCC tissues and cell lines.

Consistent with the tumor suppressive function of DAPK1, clinical studies also revealed that the expression of DAPK1 is significantly decreased in chronic lymphocytic leukemia, breast cancer and head and neck cancer $(16,28,29)$. Importantly, loss of DAPK1 expression is associated with advanced tumor stages and tumor metastasis (24). Studies of DAPK1 expression pattern and regulation mechanism in HCC are limited. Matsumoto et al investigated the expression of DAPK1 in 43 Japanese HCC patients (30). Through association study, they found DAPK1-negative HCC cases were associated with high serum AFP, lower tumor differentiation, and less apoptosis. However, while they revealed that the status of DAPK1 protein expression correlated with IFN- $\gamma$-receptor and Fas expression, but not the promoter methylation status, how IFN- $\gamma$-receptor and Fas control DAPK1 expression in HCC was not addressed.

In our study, by utilizing 70 pairs of Chinese HCC specimens, DAPK1 was found to be remarkably reduced or even lost in HCC tissues by comparison with neighboring non- tumorous tissues, which is consistent with Matsumoto et al (30). Moreover, the transcriptional regulation mechanism of $D A P K 1$ has been carefully studied through luciferase assay and ChIP experiment. Two tandem NF- $\kappa \mathrm{B}$ binding sites locating in -190 to -181 bp and -176 to -167 bp of DAPK1 promoter could be recognized by BRMS1, and responsible for BRMS1-mediated transcriptional activation. It has been noted that NF- $\kappa \mathrm{B}$ is an important modification substrate of BRMS1·mSin3a.HDAC complex (10). BRMS1 suppresses several metastasis-related genes through deacetylating $\mathrm{NF}-\kappa \mathrm{B}$ subunits $(21,22,31)$. However, Shanmugam et al recently reported that $\mathrm{NF}-\kappa \mathrm{B}$ was able to bind to DAPK1 promoter together with HDACs and played a negative role in regulating DAPK1 expression in acute myeloid leukemia (32). More interestingly, Li et al found that the cell cycle regulator ING4 was specifically induced by BRMS1 through suppressing NF- $\kappa$ B activities as well, because $\mathrm{NF}-\kappa \mathrm{B}$ functions as a transcriptional inhibitor of ING4 (33). Based on all these pieces of evidence, we speculate that BRMS1 might also be able to upregulate $D A P K 1$ expression through releasing DAPK1 promoter from the negative regulation of $\mathrm{NF}-\kappa \mathrm{B}$. Further experiments are in progress to demonstrate this hypothesis and elucidate the underlying molecular mechanism. It would also be important and interesting to investigate how BRMS1 and DAPK1 collaborate to regulate tumor metastasis.

\section{Acknowledgements}

We thank Dr Hexige Saiyin (Fudan University, China) for the kind help in analyzing tissue microarray. We also thank Dr Xuechao Wan (Fudan University, China) for his helpful discussion. This study was supported by the National Natural Science Foundation of China (31000558) and Zhuoxue Program of Fudan University.

\section{References}

1. Tang Z-Y, Ye S-L, Liu Y-K, Qin LX, Sun HC, Ye QH, Wang L, Zhou J, Qiu SJ, Li Y, et al: A decade's studies on metastasis of hepatocellular carcinoma. J Cancer Res Clin Oncol 130: 187-196, 2004.

2. Fidler IJ: The pathogenesis of cancer metastasis: The 'seed and soil' hypothesis revisited. Nat Rev Cancer 3: 453-458, 2003.

3. Seraj MJ, Samant RS, Verderame MF and Welch DR: Functional evidence for a novel human breast carcinoma metastasis suppressor, BRMS1, encoded at chromosome 11q13. Cancer Res 60: 2764-2769, 2000.

4. Shevde LA, Samant RS, Goldberg SF, Sikaneta T, Alessandrini A, Donahue HJ, Mauger DT and Welch DR: Suppression of human melanoma metastasis by the metastasis suppressor gene, BRMS1. Exp Cell Res 273: 229-239, 2002.

5. Smith PW, Liu Y, Siefert SA, Moskaluk CA, Petroni GR and Jones DR: Breast cancer metastasis suppressor 1 (BRMS1) suppresses metastasis and correlates with improved patient survival in non-small cell lung cancer. Cancer Lett 276: 196-203, 2009.

6. Nagji AS, Liu Y, Stelow EB, Stukenborg GJ and Jones DR: BRMS1 transcriptional repression correlates with $\mathrm{CpG}$ island methylation and advanced pathological stage in non-small cell lung cancer. J Pathol 221: 229-237, 2010.

7. Li J, Cheng Y, Tai D, Martinka M, Welch DR and Li G: Prognostic significance of BRMS1 expression in human melanoma and its role in tumor angiogenesis. Oncogene 30: 896-906, 2011.

8. Liu Y, Mayo MW, Nagji AS, Smith PW, Ramsey CS, Li D and Jones DR: Phosphorylation of RelA/p65 promotes DNMT-1 recruitment to chromatin and represses transcription of the tumor metastasis suppressor gene BRMS1. Oncogene 31: 1143-1154, 2012. 
9. Kodura MA and Souchelnytskyi S: Breast carcinoma metastasis suppressor gene 1 (BRMS1): Update on its role as the suppressor of cancer metastases. Cancer Metastasis Rev 34: 611-618, 2015.

10. Meehan WJ, Samant RS, Hopper JE, Carrozza MJ, Shevde LA, Workman JL, Eckert KA, Verderame MF and Welch DR: Breast cancer metastasis suppressor 1 (BRMS1) forms complexes with retinoblastoma-binding protein 1 (RBP1) and the mSin3 histone deacetylase complex and represses transcription. J Biol Chem 279: 1562-1569, 2004

11. Wu Y, Jiang W, Wang Y, Wu J, Saiyin H, Qiao X, Mei X, Guo B, Fang $\mathrm{X}$, Zhang L, et al: Breast cancer metastasis suppressor 1 regulates hepatocellular carcinoma cell apoptosis via suppressing osteopontin expression. PLoS One 7: e42976, 2012.

12. Wu J, Wang Y, Qiao X, Saiyin H, Zhao S, Qiao S and Wu Y: Cloning and characterization of a novel human BRMS1 transcript variant in hepatocellular carcinoma cells. Cancer Lett 337: 266-275, 2013.

13. Bialik S and Kimchi A: The death-associated protein kinases: Structure, function, and beyond. Annu Rev Biochem 75: 189-210, 2006.

14. Bialik S and Kimchi A: The DAP-kinase interactome. Apoptosis 19: 316-328, 2014.

15. Yegnasubramanian S, Kowalski J, Gonzalgo ML, Zahurak M, Piantadosi S, Walsh PC, Bova GS, De Marzo AM, Isaacs WB and Nelson WG: Hypermethylation of $\mathrm{CpG}$ islands in primary and metastatic human prostate cancer. Cancer Res 64: 1975-1986, 2004.

16. Raval A, Tanner SM, Byrd JC, Angerman EB, Perko JD, Chen SS, Hackanson B, Grever MR, Lucas DM, Matkovic JJ, et al: Downregulation of death-associated protein kinase 1 (DAPK1) in chronic lymphocytic leukemia. Cell 129: 879-890, 2007.

17. Benderska $\mathrm{N}$ and Schneider-Stock R: Transcription control of DAPK. Apoptosis 19: 298-305, 2014.

18. Gao Q, Qiu SJ, Fan J, Zhou J, Wang XY, Xiao YS, Xu Y, Li YW and Tang $\mathrm{ZY}$ : Intratumoral balance of regulatory and cytotoxic $\mathrm{T}$ cells is associated with prognosis of hepatocellular carcinoma after resection. J Clin Oncol 25: 2586-2593, 2007.

19. Boyd KE, Wells J, Gutman J, Bartley SM and Farnham PJ: c-Myc target gene specificity is determined by a post-DNAbinding mechanism. Proc Natl Acad Sci USA 95: 13887-13892, 1998

20. Cicek M, Fukuyama R, Cicek MS, Sizemore S, Welch DR, Sizemore N and Casey G: BRMS1 contributes to the negative regulation of uPA gene expression through recruitment of HDAC1 to the NF-kappaB binding site of the uPA promoter. Clin Exp Metastasis 26: 229-237, 2009.

21. Cicek M, Fukuyama R, Welch DR, Sizemore N and Casey G: Breast cancer metastasis suppressor 1 inhibits gene expression by targeting nuclear factor-kappaB activity. Cancer Res 65 : 3586-3595, 2005.
22. Samant RS, Clark DW, Fillmore RA, Cicek M, Metge BJ, Chandramouli KH, Chambers AF, Casey G, Welch DR and Shevde LA: Breast cancer metastasis suppressor 1 (BRMS1) inhibits osteopontin transcription by abrogating NF-kappaB activation. Mol Cancer 6: 6, 2007.

23. Deiss LP, Feinstein E, Berissi H, Cohen O and Kimchi A: Identification of a novel serine/threonine kinase and a novel $15-\mathrm{kD}$ protein as potential mediators of the gamma interferoninduced cell death. Genes Dev 9: 15-30, 1995.

24. Chen HY, Lee YR and Chen RH: The functions and regulations of DAPK in cancer metastasis. Apoptosis 19: 364-370, 2014.

25. Inbal B, Cohen O, Polak-Charcon S, Kopolovic J, Vadai E, Eisenbach L and Kimchi A: DAP kinase links the control of apoptosis to metastasis. Nature 390: 180-184, 1997.

26. Kuo JC, Wang WJ, Yao CC, Wu PR and Chen RH: The tumor suppressor DAPK inhibits cell motility by blocking the integrinmediated polarity pathway. J Cell Biol 172: 619-631, 2006.

27. Chen HY, Lin YM, Chung HC, Lang YD, Lin CJ, Huang J, Wang WC, Lin FM, Chen Z, Huang HD, et al: miR-103/107 promote metastasis of colorectal cancer by targeting the metastasis suppressors DAPK and KLF4. Cancer Res 72: 3631-3641, 2012.

28. Levy D, Plu-Bureau G, Decroix Y, Hugol D, Rostène W, Kimchi A and Gompel A: Death-associated protein kinase loss of expression is a new marker for breast cancer prognosis. Clin Cancer Res 10: 3124-3130, 2004.

29. Sanchez-Cespedes M, Esteller M, Wu L, Nawroz-Danish H, Yoo GH, Koch WM, Jen J, Herman JG and Sidransky D: Gene promoter hypermethylation in tumors and serum of head and neck cancer patients. Cancer Res 60: 892-895, 2000.

30. Matsumoto H, Nagao M, Ogawa S, Kanehiro H, Hisanaga M, Ko S, Ikeda N, Fujii H, Koyama F, Mukogawa T, et al: Prognostic significance of death-associated protein-kinase expression in hepatocellular carcinomas. Anticancer Res 23B: 1333-1341, 2003.

31. Liu Y, Mayo MW, Xiao A, Hall EH, Amin EB, Kadota K, Adusumilli PS and Jones DR: Loss of BRMS1 promotes a mesenchymal phenotype through NF- $\mathrm{BB}$-dependent regulation of Twist1. Mol Cell Biol 35: 303-317, 2015.

32. Shanmugam R, Gade P, Wilson-Weekes A, Sayar H, Suvannasankha A, Goswami C, Li L, Gupta S, Cardoso AA, Al Baghdadi T, et al: A noncanonical Flt3ITD/NF-kappaB signaling pathway represses DAPK1 in acute myeloid leukemia. Clin Cancer Res 18: 360-369, 2012.

33. Li J and Li G: Cell cycle regulator ING4 is a suppressor of melanoma angiogenesis that is regulated by the metastasis suppressor BRMS1. Cancer Res 70: 10445-10453, 2010. 